\title{
Benefícios da Terapia Neuromotora Intensiva (TNMI) para o Controle do Tronco de Crianças com Paralisia Cerebral
}

\author{
Benefits of Intensive Neuromotor Therapy (INMT) for Trunk Control of Children with Cerebral Palsy
}

\begin{abstract}
Eduardo Borba Neves', Eddy Krueger², Stéphani de Pol, Michelle Cristine Neiro de Oliveira ${ }^{4}$, Armando Fábio Szinke ${ }^{5}$, Marcelo de Oliveira Rosário ${ }^{6}$
\end{abstract}

\begin{abstract}
RESUMO
Estudos científicos ainda questionam se a fisioterapia intensiva associada ao uso da roupa com elásticos (suit-órteses dinâmicas) produzem ou não desfechos benéficos para indivíduos com déficits neurológicos. Objetivo. Determinar os ganhos na função motora de tronco de crianças com diagnóstico de paralisia cerebral submetidas à cinco semanas de Terapia Neuromotora Intensiva (TNMI) com uso do traje do PediaSuit. Trata-se de um estudo observacional longitudinal de caráter quantitativo. Resultados. Foram avaliadas 22 crianças de 03 a 08 anos de idade, com déficitsneuromotores relacionados a paralisia cerebral. Foram utilizados dois instrumentos de medida: A escala GMFM 88 e um sistema de eletrogoniometriawi-fi (com sensores sem fio) denominado Biofeed ${ }^{\circledast}$ (Biosmart ${ }^{\odot}$ ). Os resultados do GMFM mostraram diferença estatística $(\mathrm{p}<0,05)$ para as dimensóes $\mathrm{A}$, Be Total (média das dimensões avaliadas) entre os escores Pré e Pós TNMI. No mesmo sentido, a eletrogoniometriawi- $f$ i apontou redução média (Pós - Pré) de 7,70" e 5,73" para o tempo de oscilação do tronco fora dos $4^{\circ}$ e $10^{\circ}$, respectivamente, e ambos com $\mathrm{p}<0,05$. Conclusáo. Conclui-se que a TNMI associada ao uso do suit (órtese dinâmica) pode promover melhoras significativas no controle de tronco de crianças com diagnóstico de paralisia cerebral.
\end{abstract}

Unitermos. Paralisia Cerebral, Reabilitação, Terapia Intensiva, Fisioterapia.

Citaçáo. Neves EB, Krueger E, Pol S, Oliveira MCN, Szinke AF, Rosário MO. Benefícios da Terapia Neuromotora Intensiva (TNMI) para o Controle do Tronco de Crianças com Paralisia Cerebral.

\begin{abstract}
Scientific studies also question whether intensive physiotherapy associated with the use of clothing with elastic (suit - dynamic orthoses) or not produce beneficial outcomes for individuals with neurological deficits. Objective. The aim of this study was to determine the gains in motor function trunk of children diagnosed with cerebral palsy underwent five weeks of Intensive Neuromotor Therapy (INMT) using suit of Pediasuit protocol. This is an observational longitudinal quantitative study. Results. We evaluated 22 children 03-08 years of age with neuromotor deficits related to cerebral palsy. Two instruments were used to measure: the scale GMFM 88 and a system of electrogoniometrywi-fi (wireless sensors) called Biofeed ${ }^{\circledast}$ (Biosmart ${ }^{\circledast}$ ). The results of the GMFM showed statistically significant difference $(\mathrm{p}<0.05)$ for the dimensions A, B and Total (average of all evaluated dimensions) scores between Pre and Post TNMI. Likewise, electrogoniometrywi-fi pointed average reduction (Post - Pre) of 7.70" and $5.73^{\prime \prime}$ for the time of oscillation of the trunk out for $4^{\circ}$ and $10^{\circ}$, respectively, and both with $\mathrm{p}<0.05$. Conclusion. We conclude that the INMT associated with the use of the suit (dynamicsorthesis) can promote significant improvements in trunk control in children with cerebral palsy.
\end{abstract}

Keywords. Cerebral Palsy, Rehabilitation, Intensive care, Physical Therapy Specialty.

Citation. Neves EB, Krueger E, Pol S, Oliveira MCN, Szinke AF, Rosário MO. Benefits of Intensive Neuromotor Therapy (INMT) for Trunk Control of Children with Cerebral Palsy.

\footnotetext{
Trabalho realizado no Centro de Pesquisa Vitória, Curitiba-PR, Brasil. 1.Fisioterapeuta, Doutor em Engenharia Biomédica. Professor do Programa de Pós-graduação em Engenharia Biomédica da Universidade Tecnológica Federal do Paraná (UTFPR), Curitiba-PR, Brasil.

2.Fisioterapeuta, Doutorando em Engenharia Biomédica pela Universidade Tecnológica Federal do Paraná (UTFPR), Curitiba-PR, Brasil. Bolsista CNPq. 3.Acadêmica do curso de Fisioterapia do Centro Universitário Campos de Andrade, Curitiba-PR, Brasil.

4.Fisioterapeuta do Centro de Pesquisas Vitória, Centro Universitário Campos de Andrade, Curitiba-PR, Brasil.

5.Fisioterapeuta do Centro de Pesquisas Vitória, Centro Universitário Campos de Andrade, Curitiba-PR, Brasil.

6.Fisioterapeuta, Especialista em Neurologia, Docente do Centro Universitário Campos de Andrade, Curitiba-PR, Brasil.
}

Endereço para correspondência: Eduardo Borba Neves Programa de Pós-graduação em Engenharia Biomédica (PPGEB) Universidade Tecnológica Federal do Paraná (UTFPR) Av. Sete de Setembro 3165, Rebouças CEP 80230-901, Curitiba-PR, Brasil.

E-mail: borbaneves@hotmail.com 


\section{INTRODUÇÃO}

O desenvolvimento motor normal da criança é dependente da maturação do Sistema Nervoso Central (SNC). Desse modo, o período que compreende os primeiros anos de vida são de grande importância para o desenvolvimento da criança, pois é neste período que a plasticidade neural demonstra maior potencial ${ }^{1,2}$.

O desenvolvimento motor acontece gradativamente, com a aquisição do controle voluntário de novos movimentose funções cada vez mais complexas, numa integração sensorial e motora que evolui no sentidocéfalo-caudal e proximo-distal ${ }^{3}$. Isso pode ser observado no desenvolvimento de uma criança normal, pois antes de adquirir a capacidade da fala, ela se comunica com o ambiente por meio dosmovimentos corporais. Dessa maneira, por meio de estímulos sensoriais e motores, a criança ganhagradativamente o domínio corporal com consequente incremento nas capacidades de bipedestação e deambulaçãó .

Durante o primeiro ano de vida, os comportamentos motores são o principal mecanismo de aprendizado da criança, todo o movimento é uma oportunidade para adquirir conhecimento sobre o ambiente, estimulando assim também o desenvolvimento cognitivo. Ao nascimento o lactante tem a capacidade de virar-se em direção ao som da voz de sua mãe e visualizar objetos próximos a sua face. Esses estímulos auditivos e visuais fazem o lactante desafiar o seu sistema motor ${ }^{5}$ estimulando áreas no córtex cerebral fazendo com que haja um processo gradativo de maturação. Aumentando assim o nível de mielinização cortical, possibilitando o ganho no controle motor ${ }^{1}$.

Estudos indicam ${ }^{1-4}$ que os comportamentos motores nem sempre surgem em uma sequência exata, e que nem todos os indivíduos adquirem as mesmas habilidades na mesma idade cronológica. Isso se explica pelo fato que a evolução é determinada tanto por fatores genéticos como também ambientais.

Uma das patologias mais frequentes e que afeta diretamente o controle motor é a Paralisia Cerebral (PC) (também denominada encefalopatia crônica não progressiva). As crianças acometidas pela PC podem apresentar distúrbios sensoriais, cognitivos, de comunicação, de percepção, de comportamento, como desordens epiléticas, padrôes anormais de postura e alteração do tônus postu- $\mathrm{ral}^{6}$. Alguns protocolos de fisioterapia intensiva associada ao uso de suits (órtese dinâmica em forma de trajes) têm sido indicados ao tratamento dessas crianças, entre eles, o PediaSuit, o TheraSuit, o PinguinSuit, e o AdeliSuit ${ }^{7-9}$. O suit é um traje, normalmente, composto por: colete, short, joelheiras e calçados adaptados com ganchos e cordas elásticas que ajudam a posicionar o corpo num alinhamento físico adequado?.

A recomendação dos trajes se justificam, pois, normalmente, a criança com PC apresenta hipotonia axial (principalmente de tronco) e espasticidade na musculatura do esqueleto apendicular (membros) ${ }^{10}$. Por isso, o controle adequado do tronco é um dos principais aspectos envolvidos no processo de diagnóstico/reabilitação de pacientes neurológicos. A função dos músculos do tronco é um fator essencial para atividades como sentar e engatinhar ${ }^{11}$. Assim, o controle do tronco e do equilíbrio na posição sentada e em ortostatismo são habilidades essenciais para que a criança possa adquirir autonomia na realização de movimentos funcionais ${ }^{10}$.

Por outro lado, Frange et al. ${ }^{8}$ concluem sua revisão sistemática sobre a fisioterapia intensiva associada ao uso da roupa com elásticos (como no Pediasuit) afirmando que ainda não é possível determinar se esses métodos produzem ou não desfechos benéficos para indivíduos com déficits neurológicos. Neste sentido, o presente estudo tem por objetivo determinar os ganhos na função motora de tronco de crianças com diagnóstico de paralisia cerebral submetidas acinco semanas de Terapia Neuromotora Intensiva (TNMI) com uso do traje do PediaSuit.

\section{MÉTODO}

O protocolo desta pesquisa foi submetido ao Comitê de Ética em pesquisa do Centro Universitário Campos de Andrade e aprovado sob o no 000404/11. Os responsáveis de todas as crianças avaliadas assinaram os termos de consentimento livre e esclarecido em duas vias. Trata-se de um estudo observacional longitudinal de caráter quantitativo. $\mathrm{O}$ protocolo de tratamento aplicado às crianças foi a TNMI que é uma variação do protocolo denominado PediaSuit?

O estudo foi realizado no Centro de Pesquisas Vitória, com o consentimento dos responsáveis, durante os módulos de tratamento regular das crianças. Neste 
estudo, foram avaliadas 22 crianças da regiâa metropolitana de Curitiba - PRde 03 a 08 anos de idade, com déficitsneuromotores relacionados a problemas ocorridos no período gestacional ou logo após o nascimento (PC $)^{6}$. A amostra foi bastante heterogênea, com comprometimentos motores diversos, classificadas pelo Gross Motor Function Classification System (GMFCS) entre os níveis II (Anda com limitaçôes) e V (Transportado por cadeira de rodas manual).

O protocolo de tratamento compreendeu as seguintes atividades, distribuídas de acordo com a necessidade e objetivo do tratamento de cada criança: (a) Aquecimento (incluindo massagem, alongamento, cinesioterapia com mobilização passiva, ativa assistida e ativa); (b) Cinesioterapia (gaiola com cinesioterapia ativo resistido); (c) Cinesioterapia com uso do PediaSuit (gaiola com os elásticos, prancha de equilíbrio, bola, rolos, feijão, cama elástica); (d) Manobras de fisioterapia respiratória; (e) Treino de marcha (com uso do suit em terrenos irregulares, escada, rampa, esteira, grama, barras paralelas sem e com obstáculos); (f) Atividades de motricidade fina (uso de massinha, desenho com lápis, pintura com pincel, colagem, jogos de encaixe, manipulação de objetos e brinquedos); (g) osteopatia crânio-sacral; e (h) kinesiotape (bandagem funcional).

As avaliaçooes foram realizadas em dois momentos distintos: (1) no terceiro dia de tratamento; (2) no último dia de tratamento. A opção por fazer a primeira avaliação no terceiro dia de tratamento teve por objetivo propiciar um tempo de adaptação paciente-terapeuta que permitisse a criança se sentir familiarizacom os terapeutas e com o ambiente. O protocolo de tratamento foi composto por 75 horas de TNMI divididas em cinco semanas, com três horas diárias e finais de semana livres.

Para avaliação dos ganhos obtidos no controle do tronco das crianças submetidas à TNMI, foram utilizados dois instrumentos de medida. A escala GMFM 88 e um sistema de eletrogoniometriawi-fi (com sensores sem fio) denominado Biofeed ${ }^{\circledast}$ (Biosmart $^{\oplus}$ ). Ambos os instrumentos foram aplicados em dia único, por um fisioterapeuta experiente familiarizado com o método, em uma sala confortável e ampla, com chão de superfície lisa e firme. As crianças trajavam roupas leves, sem sapatos e a temperatura da sala foi mantida de $22^{\circ}$ a $24^{\circ} \mathrm{C}$.
O GMFM 88 é pontuado em uma escala de 0 a 3 (0,1,2 e 3), onde: a pontuação 0 deve ser atribuída quando a criança não inicia o movimento;a pontuaçáo 1 a criança que inicia o movimento e realiza menos de $10 \%$ da atividade; a pontuaçáo 2 quando a criança realiza parcialmente, ou seja, entre $10 \%$ e menos de $100 \%$ do movimento; e a pontuação 3 para a criança que realiza totalmente o movimento (100\%). Qualquer item não testado (NT) deve ser pontuado com o valor 0. Para obter o resultado final, o escore é transformado em percentil e após o resultado final no escore geral do teste é obtido pelo somatório dos resultados divididos pelo número de dimensôes testadas ${ }^{12}$.

O Biofeed $^{\oplus}\left(\right.$ Biosmart $\left.^{\oplus}\right)$ é um equipamento que permite o registro dos movimentos corporais realizados em diversas posturas, podendo ser estáticas ou dinâmicas $^{13,14}$. A frequência de aquisição é de $15 \mathrm{~Hz}$, o que dá uma boa precisão na aquisição dos dados. A colocação dos sensores foi sempre na regiáo anterior do tronco, no manúbrio do osso esterno. A análise consiste em registrar por meio de gráficos gerados pelo Biofeed ${ }^{\oplus}$ no plano coronal anterior e posterior. Para o registro inicial, o paciente foi posicionado sentado, com as coxas fixadas por ataduras elásticas ao assento, visando minimizar movimentos que pudessem modificar o ponto de apoio. O sensor foi fixado ao tórax, sobre o osso esterno por atadura de tecido (Figura 1).Quanto ao tempo de coleta dos dados, foi utilizadaa janela central dos tempos propostos por Neves et al. ${ }^{15}$ (30, 60 e 90 s), com o tempo de 60 s.

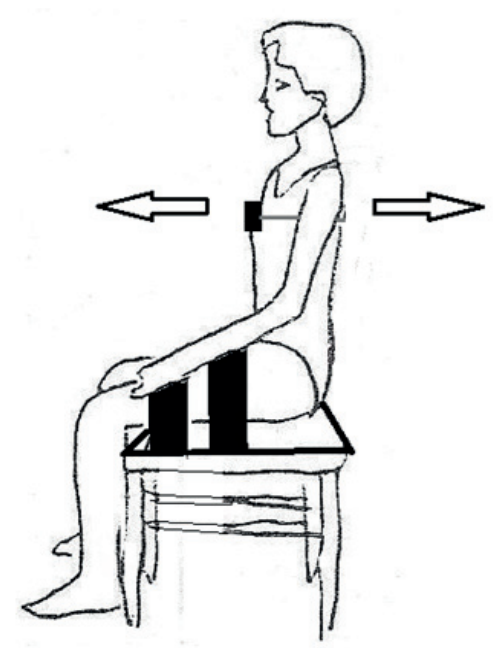

Figura 1. Ilustração do protocolo de aquisição de dados por meio do eletrogoniometrowi-fi Biofeed $^{\oplus}$ (Biosmart $^{\circledR}$ ). 
Para a análise dos padrốes de oscilação do tronco, foram verificados os tempos de oscilação do tronco fora das duas janelas de normalidade: (1) a proposta por Gagey e Weber ${ }^{16}$ que considera normal o equilíbrio estático entre $0^{\circ}$ e $4^{\mathrm{o}}$; e (2) a proposta por Moffate et al. ${ }^{17}$ que admite a normalidade para valores de $0^{\circ}$ a $10^{\circ}$.

Para análise dos dados foi utilizada a estatística descritiva, o coeficiente de correlação de Pearson; o teste t para amostras independentes e o teste t pareado. Todos os cálculos foram realizados no Statistical Package for Social Science for Windows versão 17.0 (SPSS 17.0).

\section{RESULTADOS}

As 22 crianças avaliadas apresentavam os seguintes dados antropométricos médios: idade $(4,36 \pm 1,71$ anos de idade); peso $(13,72 \pm 3,84 \mathrm{~kg})$; e altura $(103,68 \pm 11,59$ $\mathrm{cm})$. A Tabela 1 apresenta os resultados das avaliaçóes (Pré e Pós 5 semanas de TNMI) realizados com a escala GMFM e com o Biofeed ${ }^{\oplus} \mathrm{e}$ do teste $\mathrm{t}$ para amostras pareadas. Para a análise dessa tabela é importante destacar que das 22 crianças avaliadas: todas apresentaram escores válidos nas dimensôes A e B do GMFM; sete para a dimensão C; e apenas três para as dimensóes D e E. Todas as crianças avaliadas compareceram a todos os dias de tratamento.

A Tabela 2 apresenta as correlaçóes encontradas entre os dois momentos de avaliaçấo. Esses valores podem ser utilizados para avaliar a confiabilidade e reprodutibilidade dos processos de medição utilizados na coleta dos dados.

\section{DISCUSSÃO}

A principal teoria por trás da melhora do padrão motor pela terapia intensiva com o uso de trajes especiais (suits) é que a sua utilização induz um forte estímulo aferente sobre o sistema nervoso, principalmente o proprioceptivo, buscando recuperar o atraso motor decorrente da $\mathrm{PC}^{18}$. Essa hipótese pode ser confirmada pelos ganhos no controle de tronco adquiridos por eletrogoniometria e mostrados na Tabela 1 por meio da redução média (pós pré) de 7,70" e 5,73" para o tempo de oscilação do troncofora dos $4^{\circ}$ e $10^{\circ}$ respectivamente (mesmo que esses valores ainda estejam fora dos padróes normais). A aquisição da postura é obtida por volta do $3^{\circ}$ mês, essa aquisição e manutenção da postura dependem do aprendizado

Tabela 1

Resultados das avaliações de 22 crianças com paralisia cerebral, Pré e Pós 5 semanas de TNMI, Curitiba-PR, Brasil, 2012

\begin{tabular}{|c|c|c|c|c|c|}
\hline Variável & Média & $\begin{array}{l}\text { Desvio } \\
\text { Padráo }\end{array}$ & $\mathbf{N}$ & $\mathbf{t}$ & p valor \\
\hline GMFM Dimensāo A Pré TNMI (\%) & 56,14 & 27,17 & \multirow{2}{*}{22} & \multirow{2}{*}{$-6,89$} & \multirow{2}{*}{0,001} \\
\hline GMFM Dimensão A Pós TNMI (\%) & 66,96 & 25,53 & & & \\
\hline GMFM Dimensāo B Pré TNMI (\%) & 31,41 & 23,52 & \multirow{2}{*}{22} & \multirow{2}{*}{$-3,63$} & \multirow{2}{*}{0,002} \\
\hline GMFM Dimensão B Pós TNMI (\%) & 35,88 & 24,75 & & & \\
\hline GMFM Dimensão C Pré TNMI (\%) & 8,73 & 21,71 & \multirow{2}{*}{07} & \multirow{2}{*}{$-2,08$} & \multirow{2}{*}{0,050} \\
\hline GMFM Dimensão C Pós TNMI (\%) & 11,27 & 26,81 & & & \\
\hline GMFM Dimensão D Pré TNMI (\%) & 3,27 & 10,18 & \multirow{2}{*}{03} & \multirow{2}{*}{$-1,62$} & \multirow{2}{*}{0,120} \\
\hline GMFM Dimensão D Pós TNMI (\%) & 5,00 & 14,98 & & & \\
\hline GMFM Dimensão E Pré TNMI (\%) & 1,14 & 5,11 & \multirow{2}{*}{03} & \multirow{2}{*}{$-1,60$} & \multirow{2}{*}{0,125} \\
\hline GMFM Dimensão E Pós TNMI (\%) & 2,73 & 8,02 & & & \\
\hline GMFM Total Pré TNMI (\%) & 33,71 & 14,26 & \multirow{2}{*}{22} & \multirow{2}{*}{$-7,83$} & \multirow{2}{*}{0,001} \\
\hline GMFM Total Pós TNMI (\%) & 41,32 & 15,03 & & & \\
\hline Tempo de oscilação fora dos $4^{\circ}$ Pré TNMI (s) & 37,00 & 15,46 & \multirow{2}{*}{22} & \multirow{2}{*}{3,95} & \multirow{2}{*}{0,001} \\
\hline Tempo de oscilação fora dos $4^{\circ}$ Pós TNMI (s) & 29,60 & 18,81 & & & \\
\hline Tempo de oscilação fora dos $10^{\circ}$ Pré TNMI (s) & 26,86 & 18,91 & \multirow{2}{*}{22} & \multirow{2}{*}{5,57} & \multirow{2}{*}{0,001} \\
\hline Tempo de oscilaçấo fora dos $10^{\circ}$ Pós TNMI (s) & 21,13 & 19,59 & & & \\
\hline
\end{tabular}

TNMI: Terapia Neuromotora intensiva 
Tabela 2

Correlação entre as avaliaçôes, pré e pós 5 semanas de TNMI, de 22 crianças com paralisia cerebral, Curitiba-PR, Brasil, 2012

\begin{tabular}{|c|c|c|}
\hline Variáveis & Correlaçáo (r) & p valor \\
\hline GMFM (A) Pré TNMI \& GMFM (A) Pós TNMI & 0,963 & $<0,001$ \\
\hline GMFM (B) Pré TNMI \& GMFM (B) Pós TNMI & 0,973 & $<0,001$ \\
\hline GMFM (C) Pré TNMI \& GMFM (C) Pós TNMI & 0,994 & $<0,001$ \\
\hline GMFM (D) Pré TNMI \& GMFM (D) Pós TNMI & 0,994 & $<0,001$ \\
\hline GMFM (E) Pré TNMI \& GMFM (E) Pós TNMI & 0,837 & $<0,001$ \\
\hline GMFM Total Pré TNMI \& GMFM Total Pós TNMI & 0,953 & $<0,001$ \\
\hline $\begin{array}{l}\text { Tempo de oscilação fora dos } 4^{\circ} \text { Pré TNMI \& } \\
\text { Tempo de oscilaçấo fora dos } 4^{\circ} \text { Pós TNMI }\end{array}$ & 0,886 & $<0,001$ \\
\hline $\begin{array}{l}\text { Tempo de oscilação fora dos } 10^{\circ} \text { Pré TNMI \& } \\
\text { Tempo de oscilaçấo fora dos } 10^{\circ} \text { Pós TNMI }\end{array}$ & 0,969 & $<0,001$ \\
\hline
\end{tabular}

TNMI: Terapia Neuromotora intensiva

e da repetição das atividades ${ }^{19}$. $\mathrm{O}$ controle postural vai se aprimorando, e já pelo $6^{\circ}$ e $7^{\circ}$ mês a criança já é capaz de sentar por pequenos períodos com apoio de braço. Após evolui para o sentar sem apoio no $8^{\circ}$ e $9^{\circ}$ mês e finalmente entre o $12^{\circ}$ e $15^{\circ}$ mês surge a marcha ${ }^{19}$.

Corroborando os resultados observados na eletrogoniometriawi-fi, os resultados do GMFMapresentados na Tabela 1 mostram diferençasestatísticas $(\mathrm{p}<0,05)$ para as dimensōes $\mathrm{A}, \mathrm{B}$ e total (média das dimensões avaliadas) entre os escores Pré e Pós TNMI (Pares 1,2 e 6).

A dimensão B do GMFM é a mais específica para avaliar os ganhos de função no tronco, pois se refere à avaliação na posição sentada ${ }^{12}$, escopo principal deste trabalho. Assim, pode-se entender que ambos os instrumentos sugerem melhora significativa na função de tronco das crianças avaliadas no presente estudo. Destaca-se que as demais dimensóes ( $\mathrm{C}, \mathrm{D}$ e $\mathrm{E}$ ) foram avaliadas apenas em sete, três e três crianças, respectivamente, o que limita a análise estatística e fragiliza as possíveis generalizaçóes sobre os resultados dessas dimensões.

No mesmo sentido, autores avaliaram ${ }^{18}$ o efeito do tratamento com um suit em 30 crianças (de 4-12 anos de idade) com diplegiaespástica decorrente de PC. As crianças foram divididas em dois grupos: experimental $(\mathrm{N}=15)$ que fizeram uso do suit e controle $(\mathrm{N}=15)$ que recebeu apenas a fisioterapia tradicional sem o uso de trajes especiais. $\mathrm{O}$ tratamento foi aplicado por duas horas diariamente (com intervalos breves de $20 \mathrm{~min}$ ) durante três semanas. Os autores observaram diferença estatística significativa $(\mathrm{p}<0,001)$ quando compararam os resultados do GMFM 88 pré e pós o tratamento tanto para o grupo experimental quanto para o grupo controle. $\mathrm{Na}$ quele estudo, a comparação entre grupos mostrou que eles não foram diferentes na avaliação pré-tratamento $(\mathrm{p}=0,08)$, no entanto apresentaram diferenças significativas $(\mathrm{p}=0,03)$ na fase pós-tratamento. Entretanto, como os dois grupos tiveram melhora percentual média de 7,52\% para o grupo experimental e $9,91 \%$ para o grupo controle, os autores náo se posicionaram quanto a efetividade do traje, apenas afirmaram que a terapia intensiva teve resultados positivos em ambos os grupos avaliados.

Por outro lado, apesar de observar-se $\mathrm{e}^{20}$ melhoras mínimas (dados descritivos) com a terapia intensiva e o uso do TheraSuitem duas crianças com PC no ano de 2010, num estudo maior, realizado em 2011, os mesmos autores ${ }^{21}$ examinaram os efeitos do traje TheraSuit completo ( $\mathrm{N}=10$, com o uso de elásticos) contra um grupo controle ( $\mathrm{N}=10$, sem o uso de elásticos) durante um programa de fisioterapia intensiva. O programa consistiu em quatro horas diárias durante cinco dias semanais durante um período de três semanas. As avaliaçôes Pediatric Evaluation of Disability Inventory (PEDI) e Gross Motor Function Measure (GMFM)-66 foram divididas em pré e dois instantes pós-tratamento ( 4 e 9 semanas após a intervenção). Os resultados não mostraram diferençasestatísticas intragrupo (pré e pós) e intergrupos (experimental e controle). Os autores concluíram que as crianças que fizeram uso do traje TheraSuit completo não apresenta- 
ram melhoras durante o tratamento nem comparando-se com as crianças do grupo controle durante o programa de fisioterapia intensiva.

Descreve-se $\mathrm{e}^{7}$ um caso de uma criança com diplegia espástica que foi tratada com o PediaSuit e, no mesmo sentido dos resultados deste estudo, os autores também observaram melhora significativa na função motora avaliada pelo GMFM, com ganhos da ordem de onze pontos percentuais nas dimensôes $\mathrm{A}, \mathrm{B}$ e Total.

Além dos resultados positivos observados com a aplicação da TNMI com o uso do traje do Pediasuit, o presente estudo destaca uma ferramenta tecnológica (a eletrogoniometriawi-fi) que pode ser bastante útil na avaliação de processos de reabilitação de pacientes com disfunçóes motoras. Como exemplo, o emprego da eletrogoniometria é amplamente utilizado no campo da Engenharia de Reabilitação, principalmente como mecanismo de biofeedback para o controle de sistemas que utilizam estimulação elétrica funcional ${ }^{22-24}$. Evidenciou$-\mathrm{se}^{14}$ a melhora de uma paciente portadora da doença de Parkinson por meio do aparelho Biofeed ${ }^{\circledR}$ com somente quatro sessóes de reabilitação física. No presente estudo essa acuidade de medição do sistema foi confirmada pelas diferenças de controle de tronco pré e pós-tratamento com TNMI.

Os resultados do presente estudo foram satisfatóriospara a melhora do padrão motor e controle de tronco das crianças afetadas por PC no grupo avaliado. No entanto, além dessa melhora motora a terapia neuromotora intensiva pode melhorar a qualidade de vida e interação social da criança. Como afirma a pesquisa onde aplicou$-\mathrm{se}^{25}$ uma terapia induzida por contenção por seis horas diárias durante dez dias em 63 crianças com PC e concluíram que a terapia intensiva acarretou em um aumento na qualidade de vida, no aspecto relaçóes sociais, das crianças estudadas.

Como limitaçôes do estudo, pode-se destacar que este descreve os resultados observados ao final de um período de cinco semanas de tratamento e, num grupo heterogêneo de crianças com distúrbios neuromotores, por isso,tais ganhos podem não perdurar após um tempo decorrido do tratamento. Assim, ressalta-se o cuidado na generalização dos resultados e a necessidade de uma ava- liação longitudinal para avaliar se a melhora alcançada se perpetua no tempo.

\section{CONCLUSÃO}

Os resultados encontrados no presente estudo sugerem que um tratamento de cinco semanas de Terapia Neuromotora Intensiva associada ao uso do suit (órtese dinâmica em forma de traje) pode promover melhoras significativas na função motora de tronco de crianças com diagnóstico de paralisia cerebral. E ainda, que a eletrogoniometriawi-fi é uma ferramenta que pode ser aplicada ao monitoramento e acompanhamento do processo de reabilitação de pacientes com déficits motores. No entanto, este tipo de tratamento (com o uso de suits) ainda possui poucos centros de reabilitação capacitadospara estesmétodos no Brasil. O métodoexige a formação específica dos terapeutas e apresenta um custo elevado para a família dos pacientes (cerca de \$3500).

\section{AGRADECIMENTOS}

Os autores ao CNPQ pelo suporte financeiro para a realização deste trabalho. Nossos agradecimentos também aos revisores pelas sugestóes para o aprimoramento deste trabalho e ao corpo editorial da revista neurociências.

\section{REFERÊNCIAS}

1.Gioda FR, Ribeiro CM. Aquisição e refinamento do Sentar independente: Um estudo transversal. Rev Bras Cineantropom Desempenho Hum 2006;8(4):91-8.

2.Martinez CMS, Joaquim RHVT, Oliveira EB, Santos IC. Suporte informacional como elemento para orientação de pais de pré-termo: um guia para o serviço de acompanhamento do desenvolvimento no primeiro ano de vida. Rev Bras Fisioter 2007;11(1):73-81.

http://dx.doi.org/10.1590/S1413-35552007000100012

3.Fonseca LA, Rodrigues AG. Estudo comparativo do desenvolvimento neuropsicomotor em crianças não portadoras do vírus HIV cujas mães tomaram AZT e filhos de mães não portadoras do vírus HIV [Trabalho de Conclusão de Curso de Graduação em Fisioterapia]. Belém-PA: Universidade da Amazônia; 2007, 59p.

4.Santos S, Dantas L, Oliveira JA. Desenvolvimento motor de crianças, de idosos e de pessoas com transtornos da coordenação. Rev Paul Educ Fís 2004;18:33-44.

5.Umphred D. Reabilitação neurológica: Elsevier Editora Ltda.; 2011, 876p.

6.Rosenbaum P, Paneth N, Leviton A, Goldstein M, Bax M, Damiano D, et al. A report: the definition and classification of cerebral palsy April 2006. Dev 
Med Child Neurol Suppl 2007;109:8-14.

7.Neves EB, Scheeren EM, Chiarello CR, Costin ACMS, Mascarenhas LPG. $\mathrm{O}$ PediaSuit ${ }^{\mathrm{TM}}$ na reabilitaçáo da diplegia espástica: um estudo de caso. Lecturas, Educación Física y Deportes (Buenos Aires) 2012;166:1-9.

8.Frange CMP, Silva TOT, Filgueiras S. Revisão Sistemática do Programa Intensivo de Fisioterapia Utilizando a Vestimenta com Cordas Elásticas. Rev Neurocienc 2012;20(4):517-26.

9.Scheeren EM, Mascarenhas LPG, Chiarello CR, Costin ACMS, Oliveira L, Neves EB. Description of the Pediasuit ProtocolTM. Fisioter Mov 2012;25(3):473-80.

http://dx.doi.org/10.1590/S0103-51502012000300002

10.Forti-Bellani CD, Castilho-Weinert LV. Desenvolvimento Motor Típico, Desenvolvimento Motor Atípico e Correlaçôes na Paralisia Cerebral. In: Castilho-Weinert LV, Forti-Bellani CD, editors. Fisioterapia em Neurologia. Curitiba-PR: Omnipax; 2011, p.350.

11.Torriani C, Queiroz SS, Cyrillo FN, Monteiro CBM, Fernandes S, Padoan $\mathrm{B}$, et al. Correlaçáo entre transferência de peso sentado e alteração sensorial em regiāo glútea em pacientes hemiplégicos/paréticos. Rev Neurocienc 2005;13(3):117-21.

12.Russell DJ, Rosenbaum PL, Avery LM, Lane M. Gross motor function measure (GMFM-66 \& GMFM-88) user's manual: Mac keith press London; 2002, 120p.

13.Alves RCA, Santos FCLd, Oliveira BT, Margi CB. Sistema de Monitoramento de Amplitude de Movimento Baseado em Redes de Sensores sem Fio Aplicado à Fisioterapia. VIII Simpósio Brasileiro de Sistemas de Informação; São Paulo-SP: Anais do VIII Simpósio Brasileiro de Sistemas de Informação; 2012; p. 504-515.

14.Cortés A, Chodur A, Gallon D, Ribas D, Machado J, Melo T, et al. Effects of biofeedback shoulder abdution in elderly with parkinsonism: a case study. Rev Neurocienc 2010;18(2):189-93.

15.Neves EB. Trends in Neuropediatric Physical Therapy. FrontPublic Health
2013;1:1-2.

16.Gagey PM, Weber B, Cunha HM, Ikeda M. Posturologia: regulação e distúrbios da posiçấo ortostática: Manole; 2000, 161p.

17.Moffat M, Bohmert JA, Hulme JB. Fisioterapia do Sistema Neuromuscular: melhores práticas /Série Physio 1ed: Guanabara Koogan; 2010, 325p.

18.Alagesan J, Shetty A. Effect of Modified Suit Therapy in Spastic Diplegic Cerebral Palsy-A Single Blinded Randomized Controlled Trial. Online J Health Allied Scs 2011;9:1-3.

19.Campos D, Santos DCC. Controle postural e motricidade apendicular nos primeiros anos de vida. Fisioter Mov 2005;18(3):71-7.

20.Bailes AF, Greve K, Schmitt LC. Changes in two children with cerebral palsy after intensive suit therapy: a case report. Ped Phys Ther 2010;22(1):76-85. http://dx.doi.org/10.1097/PEP.0b013e3181cbf224

21.Bailes AF, Greve K, Burch CK, Reder R, Lin L, Huth MM. The effect of suit wear during an intensive therapy program in children with cerebral palsy. Ped Phys Ther 2011;23(2):136.

http://dx.doi.org/10.1097/PEP.0b013e318218ef58

22.Jezernik S, Wassink RGV, Keller T. Sliding mode closed-loop control of FES: controlling the shank movement. IEEE Trans Biomed Eng 2004;51(2):263-72. http://dx.doi.org/10.1109/TBME.2003.820393

23. Gollee H, Hunt KJ, Wood DE. New results in feedback control of unsupported standing in paraplegia. IEEE Trans Neural Syst Rehabil Eng 2004;12(1):73-80.

http://dx.doi.org/10.1109/TNSRE.2003.822765

24.Fisekovic N, Popovic DB. New controller for functional electrical stimulation systems. Med Eng Phys 2001;23(6):391-9.

http://dx.doi.org/10.1016/S1350-4533(01)00069-8

25.Sakzewski L, Carlon S, Shields N, Ziviani J, Ware RS, Boyd RN. Impact of intensive upper limb rehabilitation on quality of life: a randomized trial in children with unilateral cerebral palsy. Dev MedChild Neurol 2012;54(5):415-23. http://dx.doi.org/10.1111/j.1469-8749.2012.04272.x 